\title{
Diffuse large B-cell lymphoma of tonsil and testis: unexpected metastatic sequence
}

\author{
Alexey Annenkov, Ken Nishikura, Yoichi Ajioka, \\ Motohiko Kimura, Hisanobu Shimura, Nobuhiko Nomoto
}

\begin{abstract}
Introduction: Diffuse large B-cell lymphoma is the largest subtype of non-Hodgkin's lymphomas, and is characterized by frequent extranodal presentation. The most common extranodal locations of non-Hodgkin's lymphomas are: stomach, bone, liver and testis. Simultaneous detection of multiple extranodal involvements during consultation is quite uncommon, and the majority of cases are characterized by gastric or intestinal localization. Case Report: This study has found an extranodal involvement of tonsil and testis by diffuse large B-cell lymphoma. Symptoms of tonsil lymphoma were seen one year before the same type of lymphoma appeared in the testis.
\end{abstract}

Alexey Annenkov ${ }^{1}$, Ken Nishikura ${ }^{2}$, Yoichi Ajioka ${ }^{3}$, Motohiko Kimura ${ }^{4}$, Hisanobu Shimura ${ }^{5}$, Nobuhiko Nomoto ${ }^{6}$

Authors' affiliations: ${ }^{1}$ Research fellow, Division of Molecular and Diagnostic Pathology, Niigata University Graduate School of Medical and Dental Sciences, Niigata, Japan; ${ }^{2}$ Associate professor, Division of Molecular and Functional Pathology, Niigata University Graduate School of Medical and Dental Sciences, Niigata, Japan; ${ }^{3}$ Chairman, Division of Molecular and Diagnostic Pathology, Niigata University Graduate School of Medical and Dental Sciences, Niigata, Japan; ${ }^{4}$ M.D., Department of Urology, Niigata Kobari Hospital, Niigata, Japan; ${ }^{5}$ M.D., Department of Urology, Niigata Kobari Hospital, Niigata; ${ }^{6}$ M.D., Department of Internal Medicine, Niigata Kobari Hospital, Niigata, Japan

Address corresponding to: Alexey Annenkov, Division of Molecular and Diagnostic Pathology, Niigata University Graduate School of Medical and Dental Science, Asahimachidori 1-757, Chuou-ku, Niigata, Japan, email: annenkov.a@hotmail.com

Received: 23 June 2010

Accepted: 6 August 2010

Published: 3 September 2010
Conclusion: The clinical course, similarity in the histological findings and the immunoprofile between the two organs suggested a possibility of metastatic diffuse large B-cell lymphoma from the tonsil to testis, which would be the first reported case.

Key Words: Malignant lymphoma, Tonsil, Testis, Metastasis

$* * * * * * * * *$

Annenkov A, Nishikura K, Ajioka Y, Kimura M, Shimura H, Nomoto N. Diffuse large B-cell lymphoma of tonsil and testis: Unexpected metastatic sequence. IJCRI 2010;1(1):711.

doi:10.5348/ijcri-2010-09-2-CR-2

\section{INTRODUCTION}

Diffuse large B-cell lymphoma (DLBCL) constitutes approximately $30 \%$ of all non-Hodgkin's lymphomas (NHLs) and typically presents as a rapidly enlarging symptomatic mass which is mostly due to nodal enlargement. The overall percentage of NHLs coded as being of extranodal origin is between $25 \%$ and $35 \%$ in most countries. Frequently, the origin of primary extranodal NHL can be ascribed to one given organ system or site, with most common extranodal sites being the stomach, skin, and small intestine. The breast, thyroid, lung, heart, liver, genitourinary tract, adrenal glands and bone could also be primarily affected [1].

This report details a patient with an unusual presentation of DLBCL, characterized by tonsil and 
testicular involvement, which was associated with symptoms and signs of primary involvement of both organs.

\section{CASE REPORT}

\section{Clinical findings}

On $30^{\text {th }}$ of October 2007, a 49-year-old Japanese male came to the Otorhinolaryngology Department of Niigata University General Hospital, stating that he was suffering from throat pain. The patient himself had no past history of any significant disease, however, his family history included his father suffering from Gastro-hepatic sarcoma (the precise information was not available) and his uncle suffering from lung cancer. Physical examination and laboratory tests were noncontributory to the patient's condition. On further evaluation and imaging, including Computed Tomography scan, Ultrasound examination, and Magnetic Resonance Imaging of the head and neck (Figure 1), there were no significant findings, except slightly swollen and ulcerated left tonsil. No tonsillar tissue biopsy was performed. Diagnosis of tonsillar hypertrophy was stated. The patient's throat pain disappeared for a while after medication but after 13 months the same symptoms appeared again. He visited the hospital again on $18^{\text {th }}$ of December 2008. At that time, left palatine tonsil had increased in size as compared to previous presentation and was ulcerated. The lesion was biopsied and a malignant lymphoma was suspected on histopathologic examination. On $5^{\text {th }}$ of January 2008, he underwent a partial resection of the left palatine tonsil and subsequent histopathologic examination with immunohistochemistry established the diagnosis of B-cell type malignant lymphoma. He was subsequently referred to the Internal Medicine Department of Niigata Kobari hospital for

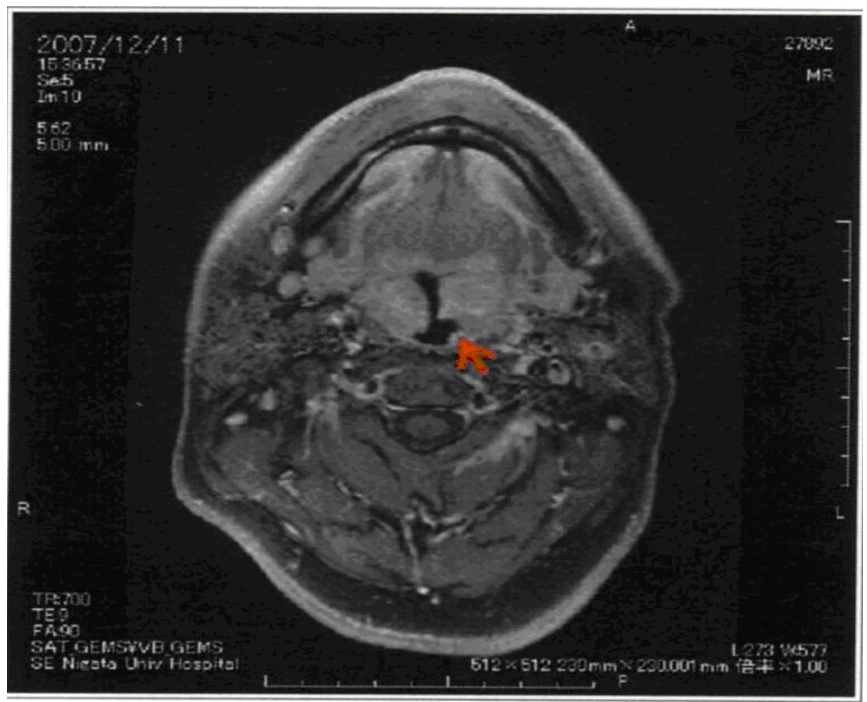

Figure 1: Magnetic resonance imaging of the head and neck. Slightly swollen tonsils with ulceration in the left tonsil indicated by red arrow. chemotherapy and was admitted to the hospital on $22^{\text {nd }}$ of January 2009.

The patient also complained of right testicular swelling since one month which was not associated with any pain for which he was referred to the Urology Department of Niigata Kobari Hospital to establish a diagnosis before starting the chemotherapy. The Computed Tomography scan and ultrasound examination revealed a solid tumor with a diameter of about $3 \mathrm{~cm}$ in his right testis. The right orchiectomy was performed on $27^{\text {th }}$ of January 2009. An immunohistological examination of the resected right testis showed malignant lymphoma looking similar to previously resected tonsillar lymphoma. This diagnosis was further supported by the fact that tumor markers were positive in the resected tonsillar tissue: IgM 30\% (normal range, 2-7\%), Kappa light chain $33 \%$ and Lambda light chain $9 \%$.

\section{Material \& Methods}

Formalin-fixed and paraffin-embedded tissue was used for routine histological procedure. Five-micrometer thick sections were stained with hematoxylin and eosin (H\&E). Sequent sections were examined immunohistochemically.

For the immunohistochemical analysis, streptavidinbiotin peroxidase method was performed using the Histofine SAB-PO (M) kit (Nichirei Biosciences Inc., Tokyo, Japan). Sections were deparaffinized, rehydrated and then microwaved at $500 \mathrm{~W}$ for seven cycles of 3 minutes in $10 \mathrm{mmol} / \mathrm{L}$ of citrate buffer $(\mathrm{pH}$ 6.0) to retrieve antigenic activity. Endogenous peroxidase activity was blocked by $0.3 \%$ hydrogen peroxide in methanol for 20 minutes. After blocking any nonspecific reaction with $10 \%$ normal rabbit serum, sections were incubated overnight at $4^{\circ} \mathrm{C}$ with antibodies (Table 1). Diaminobenzidine was used as the chromogen and sections were counterstained with hematoxylin.

\section{Pathological findings}

On gross examination, we found a yellowish-colored tumor in the testis with expansive growth of size $45 \times 40 \times 23$ $\mathrm{mm}$ with a central necrotic area measuring $27 \times 18 \mathrm{~mm}$ in size (Figure 2). On histological examination of the specimen the normal testicular parenchyma was largely replaced by diffuse infiltration of medium- to large-sized atypical lymphocytic cells without glandular structure. Neoplastic cells did not involve the spermatic cord. Vascular and lymphatic invasion by tumor cells was not seen. Tumor cells had vesicular chromatin, one or two small but distinct nucleoli, and discernible cytoplasm with high N/C ratio (Figure 3). Mitotic figures were found as high as 1 per high power field (HPF). Immunohistochemical stains demonstrated that the neoplastic cells strongly expressed markers of B-cell differentiation (CD2O and CD79a) as well as bcl-2 (Figure 5). MIB1 index was $68.2 \%$ (Table 2). From histological findings and the immunoprofile above the neoplasm was diagnosed as malignant lymphoma of diffuse large B-cell type.

The resected tonsil showed similar histological findings 


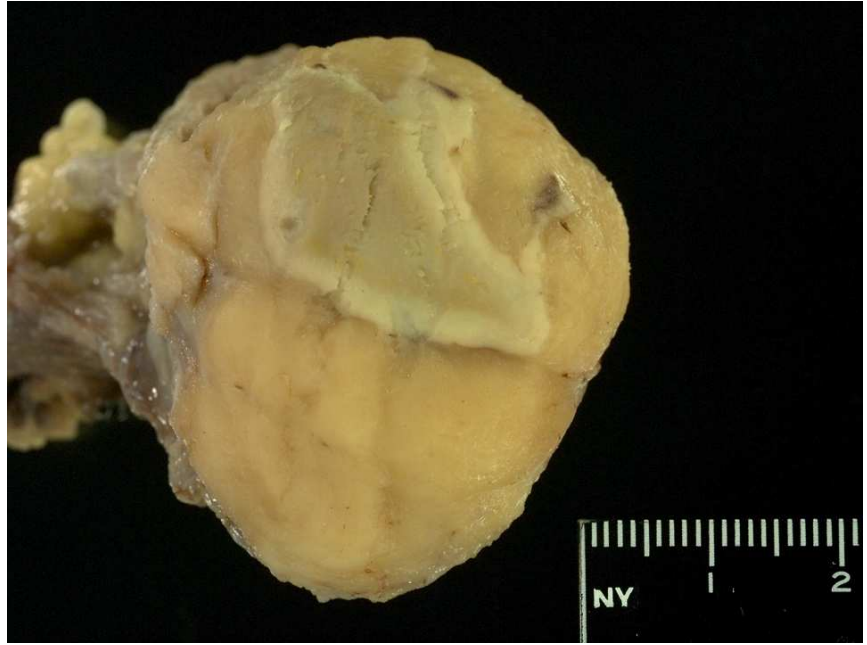

Figure 2: Macroscopic appearance of the resected right testis. A yellowish color homogeneous tumor is seen measuring $45 \times 40 \times 23$ $\mathrm{mm}$ in diameter with whitish colored zone of necrosis.

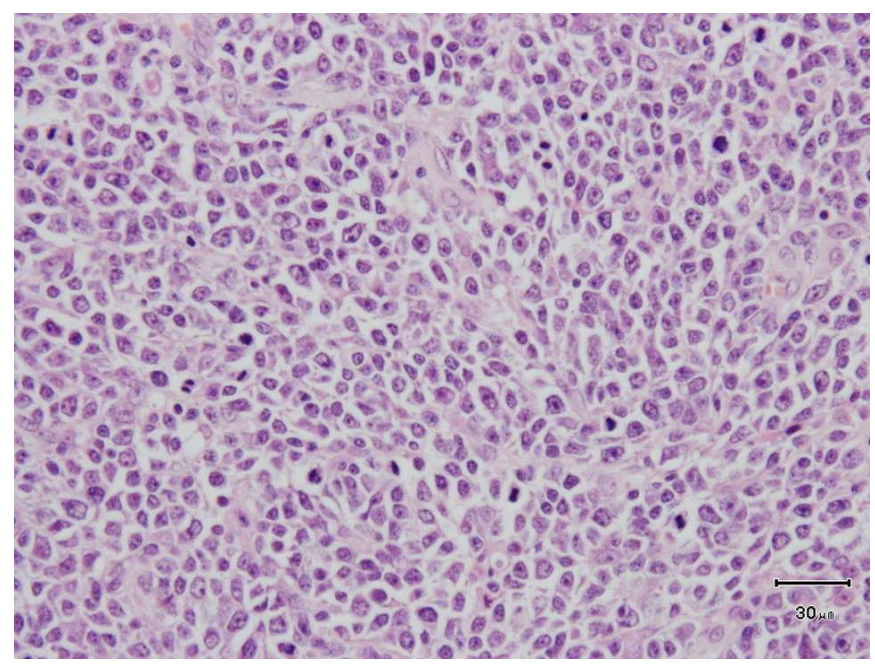

Figure 3: Microscopic appearance of the resected testis. Neoplastic medium to large sized lymphoid cells with diffuse proliferation and without glandular structure are seen. (H\&E, $\mathrm{x} 40$ )

with diffuse proliferation of atypical lymphocytic cells in sheet with frequent mitosis as 1.7/HPF (Figure 4). The tumor cells were diffusely immunoreactive for B-cell surface markers (CD20 and CD79a) and bcl-2, which was the similar to the testicular lymphoma (Figure 5). MIB1 index was $78.2 \%$.

\section{Clinical course}

The patient is presently receiving systemic chemotherapy at the Internal Medicine Department of Niigata Kobari Hospital and is doing well until now.

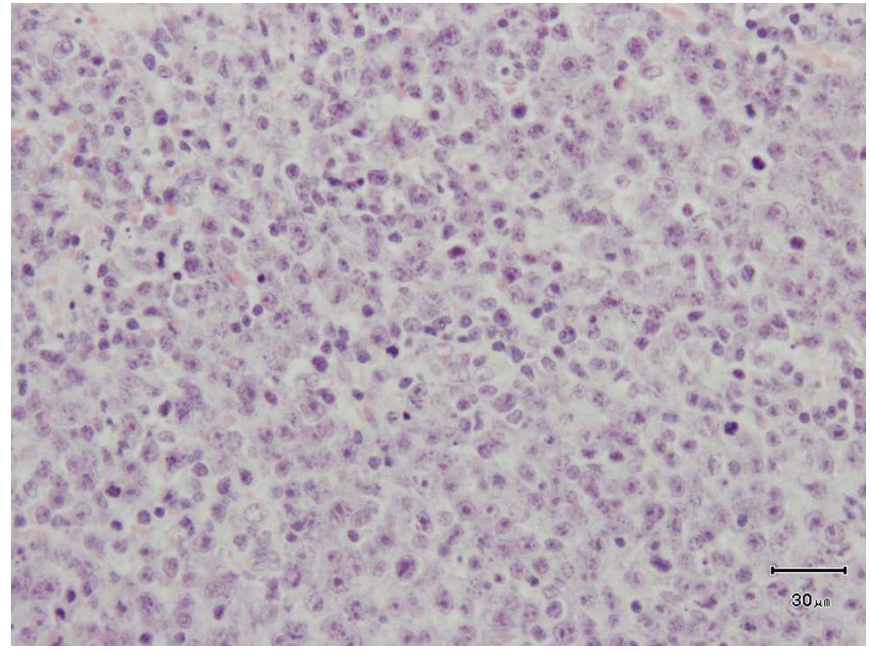

Figure 4: Microscopic appearance of the resected tonsil. Medium to large sized lymphoid cells with vesicular chromatin, one or two small nucleoli and discernible cytoplasm are seen. (H\&E, X4O)
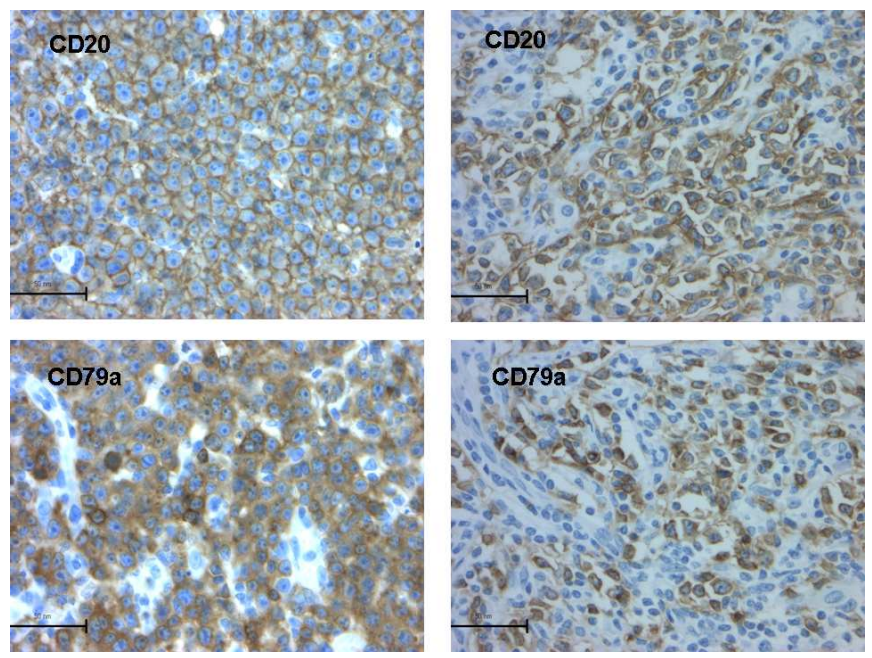

Figure 5: Expression of CD20 and CD79a immunostains in the left tonsil (left upper and lower figures) and right testis (right upper and lower figures). (x40) Both tonsil and testis strongly expressed markers of B-cell differentiation (CD20 and CD79a) on immunohistochemical staining.

\section{DISCUSSION}

The Waldeyer's ring is one of the extranodal sites which is commonly involved in DLBCL, with the most common site of involvement within the Waldeyer's ring being the tonsil. The vast majority of lymphomas in the head and neck region are B-cell type non-Hodgkin's lymphomas (NHLs). These lymphomas occur predominantly in elderly males and present themselves as a tonsillar swelling, cervical lymphadenopathy, dysphagia, odynophagia or 
Table 1: Antibodies used in immunophenotypic analyses

\begin{tabular}{cccc}
\hline Antigen & Clone & Dilution & Source \\
BCL2 & 124 & $1: 50$ & Dako, Copenhagen, Denmark \\
Cytokeratin 8/18/19 & CAM5.2 & $1: 16$ & Dako/Becton Dickinson \\
CD3 & PS1 & $1: 100$ & Novocastra, Newcasle, UK \\
CD4 & $1 \mathrm{~F} 6$ & $1: 50$ & Novocastra \\
CD5 & $4 \mathrm{C} 7$ & $1: 50$ & Novocastra \\
CD10 & $56 C 6$ & $1: 200$ & Novocastra \\
CD20 & L26 & $1: 500$ & Dako \\
CD79a & JCB117 & $1: 100$ & Nichirei Biosciences Inc., Tokyo, Japan \\
KI1(CD30) & Ber-H2 & $1: 40$ & Dako \\
KI67 & MIB1 & $1: 200$ & Dako \\
Kappa Light Chains & R10-21-F3 & $1: 100$ & Dako \\
Nambda Light Chains & N10/2 & $1: 600$ & Dako \\
\hline
\end{tabular}

Table 2: Expression of immunostains in the testicular and tonsillar lesion

\begin{tabular}{|c|c|c|}
\hline \multirow{2}{*}{ Antigen } & \multicolumn{2}{|c|}{ Expression pattern } \\
\hline & Testis & Tonsil \\
\hline BCL2 & + & + \\
\hline Cytokeratin & - & - \\
\hline CD3 & - & - \\
\hline $\mathrm{CD}_{4}$ & - & - \\
\hline CD5 & - & - \\
\hline CD10 & - & - \\
\hline CD2O & + & + \\
\hline CD79a & + & + \\
\hline $\mathrm{KI} 1(\mathrm{CD} 30)$ & - & - \\
\hline KI67 & $68.2 \%$ & $78.2 \%$ \\
\hline Kappa Light Chains & - & - \\
\hline Lambda Light Chains & - & - \\
\hline
\end{tabular}

sore throat. The majority of tonsil lymphomas are of B-cell origin and the most common histological type is diffuse large B-cell lymphoma [2-3]. On the other hand, primary non-Hodgkin's lymphoma of the testis (PTL) accounts for about $9 \%$ of testicular neoplasms and $1-2 \%$ of all NHL. It is the most common testicular malignancy in older men. Anecdotal reports have associated PTL development with trauma, chronic orchitis, cryptorchidism or filariasis exist but no case-control study has confirmed their etiologic significance. DLBCL is the most common histologic type of primary testicular lymphoma while more aggressive histologies, especially Burkitt's lymphoma being prevalent in cases of secondary involvement of the testis. The most common clinical presentation is unilateral painless scrotal swelling, sometimes associated with sharp scrotal pain or hydrocele [4]. In our patient, signs and symptoms of primary involvement in both tonsil and testis were observed.

The time span between operations due to DLBCL of both organs was shorter than one month, but the clinical picture showed us that the symptoms of malignant lymphoma of the tonsil occurred one year before that of the testis. Moreover, histopathologic findings and immunohistochemical examination confirmed similar features in both the tumors, which allowed us to consider three hypotheses about the carcinogenesis: 1) simultaneously growing tumors, 2) metastasis from tonsil to testis, and 3) metastasis from testis to tonsil. We may think about simultaneously growing tumors because of the clinical picture as both tumors showed symptoms of primary malignant lymphoma. However, primary lymphomas of testes are not associated with lymphoma elsewhere or leukemia [5]. So it is most likely a case of metastasis from one region to another. The histologic pictures of both tumors are similar to that which usually takes place in primary and metastatic tumors [6]. Furthermore testis, oropharynx and nasopharynx are derived from a common embryonic origin, the endoderm. 
On a systematic review of literature, we found that there is $5 \%$ chance of the Waldeyer's ring being involved in a cancer dissemination of PTL, although this involvement is enigmatic [7]. As it was described before, the patient complained of throat pain and had an ulcer in the left tonsil one year before the onset of testicular symptoms. This time span possibly allows us to consider metastasis of DLBCL from the tonsil to testis and can also prove our hypothesis. We could not find any reports about metastasis of DLBCL from tonsil to testis, so if our hypothesis is right it will be the first case in of its kind reported in medical literature.

\section{CONCLUSION}

In conclusion we want to highlight the second hypothesis (metastasis from tonsil to testis) because it is more suitable to the clinical picture of the disease, pathological findings and data that we have found in scientific journals during our investigation.

\section{Acknowledgements}

$* * * * * * * * *$

The authors express great thanks to Naoyuki Yamaguchi, Kazue Kobayashi and all staffs of Division of Molecular and Diagnostic Pathology, Niigata University Graduate School of Medical and Dental Sciences for their skillful technique.

\section{Author Contributions}

Alexey Annenkov - Conception and design, Acquisition of data, Analysis and interpretation of data, Drafting the article, Critical revision of the article, Final approval of the version to be published

Ken Nishikura - Conception and design, Acquisition of data, Analysis and interpretation of data, Drafting the article, Critical revision of the article, Final approval of the version to be published

Yoichi Ajioka - Analysis and interpretation of data, Critical revision of the article, Final approval of the version to be published

Motohiko Kimura - Acquisition of data, Critical revision of the article, Final approval of the version to be published Hisanobu Shimura - Acquisition of data, Critical revision of the article, Final approval of the version to be published Nobuhiko Nomoto - Acquisition of data, Critical revision of the article, Final approval of the version to be published

\section{Guarantor}

The corresponding author is the guarantor of submission.

\section{Conflict of Interest}

Author(s) declare no conflict of interest.

\section{Copyright:}

(C) Alexey Annenkov et al. 2010; This article is distributed the terms of Creative Commons attribution 3.0 License which permits unrestricted use, distribution and reproduction in any means provided the original authors and original publisher are properly credited. (Please see www.ijcasereportsandimages.com/copyright-policy.php for more information.)

\section{REFERENCES}

1. Airaghi L, Greco I, Carrabba M, Barcella M, Baldini I M, Bonara $\mathrm{P}$, et al. Unusual presentation of large $\mathrm{B}$ cell lymphoma: a case report and review of literature. Clinical and Laboratary Haematology 2006;28:338342.

2. Harabuchi $\mathrm{Y}$, Tsubota H, Ohguro S, Himi T, Asakura K, Kataura A, et al. Prognostic factors and treatment outcome in non-Hodgkin's lymphoma of Waldeyer's ring. Acta Oncology 1997;36:413-420.

3. Yamanaka N, Harabuchi Y, Sambe S, Shido F, Matsuda F, Kataura A, et al. Non-Hodgkin's lymphoma of Waldeyer's ring and nasal cavity. Clinical and immunological aspects. Cancer 1985;56:768-776.

4. Vitolo U, Ferreri AJ, Zucca E. Primary testicular lymphoma. Critical Reviews in Oncology/Hematology 2008;65:183-189.

5. World Health organization Classification of Tumours. Pathology and Genetics of Tumours of the Urinary System and Male Genital Organs. IARC Press Lyon 2004:263.

6. Economopoulos T, Papageorgiou S, Rontogianni D, Kaloutsi V, Fountzilas G, Tsatalas C, et al. Multifocal extranodal non-Hodgkin lymphoma: a clinicopathologic study of 37 cases in Greece, a Hellenic Cooperative Oncology Group study. Oncologist. 2005;10:734-738.

7. Eckert H, Smith JP. Malignant lymphoma of the testis. British Medical Journal 1963;2:891-894. 